\title{
DESIGNING FRAMEWORK OF A SEGMENTED RUBBER TRACKED VEHICLE FOR SEPANG PEAT TERRAIN IN MALAYSIA
}

\author{
ATAUR RAHMAN*, AZMI YAHAYA, MOHD. ZOHADIE, DESA AHMAD AND \\ WAN ISHAK
}

Faculty of Engineering, University Putra Malaysia,

43400, Serdang, Selangor, Malaysia

\begin{abstract}
The focus of this paper is to present the designing framework of an off-road special segmented rubber tracked vehicle that may be required to run on low bearing capacity peat terrain. To complete this study: firstly, mechanical properties of the peat terrain were first determined by using different type of apparatus. Direct shear test was performed using a Wykeham Farrance 25402 shear box apparatus to determine the internal friction angle, cohesiveness and shear deformation modulus of the peat sample. Load-sinkage test was performed using a specially made bearing capacity apparatus to determine the stiffness values of surface mat and underlying peat. Secondly, substantiate the validity of the mathematical model and finally design parameters of the special segmented rubber tracked vehicle were optimized by simulation.
\end{abstract}

Keywords: Tracked vehicle, peat terrain, tractive performance.

\section{INTRODUCTION}

In Malaysia, new requirements for greater mobility over a wide range of peat terrain, and growing demands for environmental protection and collection-transportation goods by vehicles on unprepared peat terrain constitute a significant part of the overall transportation activities. This has led to the necessity of developing segmented rubber tracked vehicle and establishing mathematical models for the vehicle-peat terrain systems, that will enable design engineers, as well as users, to evaluate a wide range of options for the selection of an optimum configuration for a given mission and environment. Various organizations in Malaysia are introduced different type of machines for performing several tasks on peat land. The machines suitabilities were justified based on the mechanical properties [2] of Sepang peat land. It was found that the engine power of all the machines are quite reasonable based on their carrying capacity. However, the tractive performances is severely affected due to the higher ground pressure distribution.

* Corresponding author 


\section{MATERIALS AND METHODS}

The feasibility of the land locomotion of the tracked vehicle operating on the weak peat terrain is discussed from the perspective of the terramechanics. Figure 1 shows the processes for designing a segmented tracked vehicle. This paper presents the discussion until the design parameter optimization technique.

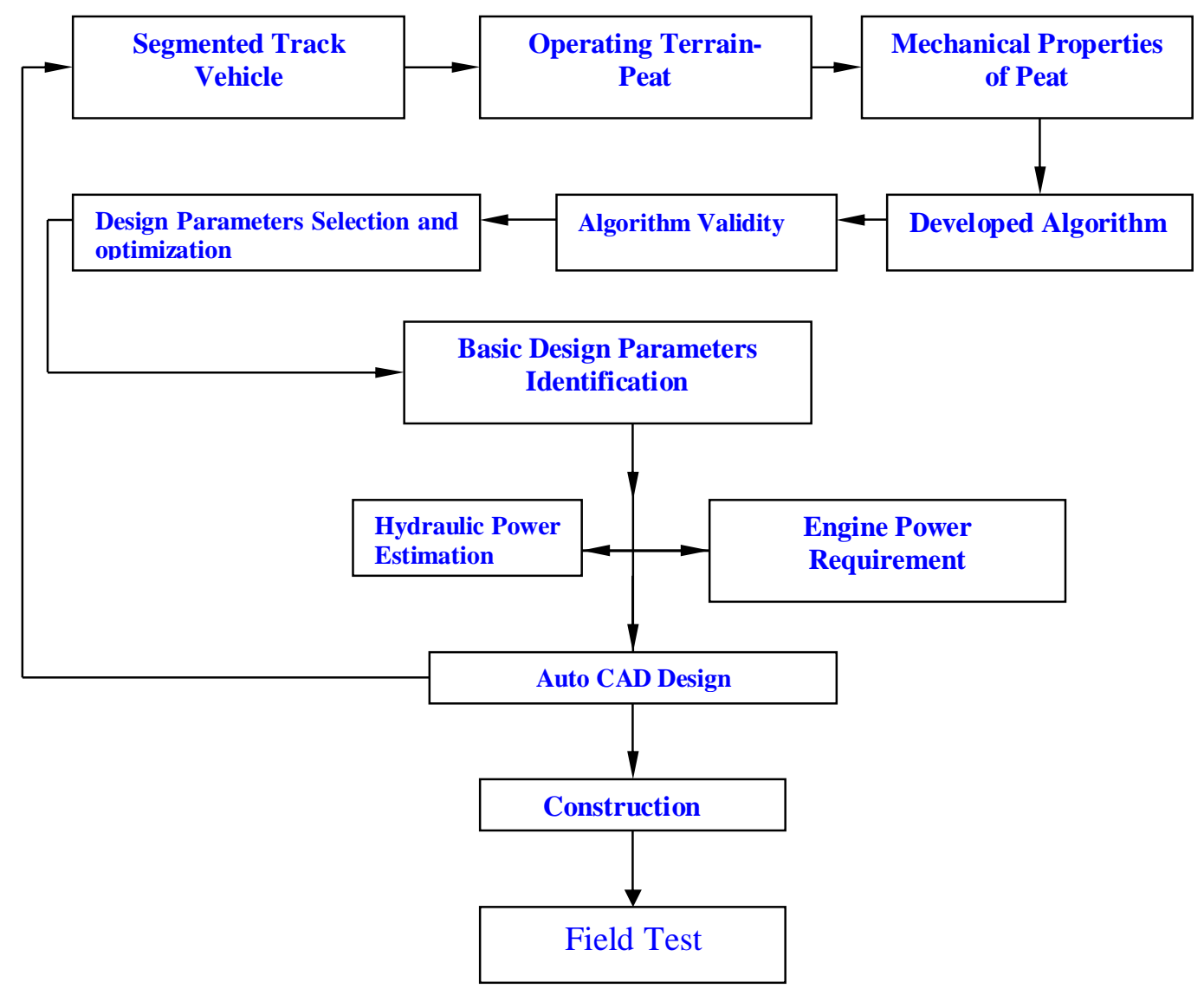

Fig. 1: Flow chart for the processes of designing a segmented tracked vehicle.

\subsection{Mechanical Properties of Peat Terrain}

To properly identify the mechanical properties of peat from a vehicle mobility viewpoint, measurement has been taken under loading conditions similar to those exerted by a vehicle on the peat terrain. Field tests were carried out at Sepang peat area, located about $45 \mathrm{~km}$ from Kuala Lumpur Malaysia for determining the mechanical properties of peat terrain including moisture content, bulk density, cohesiveness, internal friction angle, shear deformation modulus, vane shearing strength, surface mat stiffness, and underlying stiffness of peat. The mechanical properties of Sepang peat terrain including the terrain moisture content $\omega$, bulk density $\gamma_{d}$, peat surface mat stiffness $m_{m}$, underlying stiffness of peat $k_{p}$, cohesiveness $c$, internal frictional angle $\varphi$, and shear deformation modulus $K_{w}$ that are found from an earlier work [2] are given in Table 1. 
Table 1. Peat terrain parameters.

\begin{tabular}{lcccc}
\hline \multicolumn{1}{c}{ Parameters } & \multicolumn{2}{c}{ Un-drained } & \multicolumn{2}{c}{ Drained } \\
& Mean value & SD & Mean value & SD \\
\hline$\omega,(\%)$ & 83.51 & - & 79.58 & - \\
$\gamma_{\mathrm{d}},\left(\mathrm{kN} / \mathrm{m}^{3}\right)$ & 1.53 & 0.59 & 1.82 & 0.78 \\
$\left.\mathrm{c}, \mathrm{kN} / \mathrm{m}^{2}\right)$ & 1.36 & 0.21 & 2.73 & 0.39 \\
$\varphi,(\mathrm{degree})$ & 23.78 & 4.56 & 27.22 & 2.19 \\
$\mathrm{~K}_{\mathrm{w}},(\mathrm{cm})$ & 1.19 & 0.10 & 1.12 & 0.17 \\
$\mathrm{~m}_{\mathrm{m}},\left(\mathrm{kN} / \mathrm{m}^{3}\right)$ & 27.07 & 13.47 & 41.79 & 13.37 \\
$\mathrm{k}_{\mathrm{p}},\left(\mathrm{kN} / \mathrm{m}^{3}\right)$ & 224.38 & 52.84 & 356.8 & 74.27 \\
\hline
\end{tabular}

Notification: SD-Standard deviation, Source [2].

\subsection{Mathematical Model}

Consider a rigid link segmented rubber track vehicle of weight $\mathrm{W}$, track size including track ground contact length $L$, width $B$, pitch $T_{p}$, and grouser height $H$, radius of the front idler $R_{f i}$, rear sprocket $R_{r s}$, and road-wheel $R_{w}$, and height of center of gravity (C.G) $h_{c g}$, which is traversing under traction on a peat terrain at a constant speed of $v_{t}$ and applied a driving torque $Q$ at the rear sprocket by the hydraulic motor as in Fig. 10. If the pressure distribution in the track-terrain interface is assumed to be non-uniform by locating vehicle C.G rearward of the track mid point, the vehicle will traverse on the specified terrain by making an angle $\theta_{t i}$. Consequently, the track entry and exit angles at the front idler $\theta_{f i}$ and rear sprocket $\theta_{r s}$, the reaction pressure at the front idler $P_{f i}$, main straight part $P_{o}$, and rear sprocket $P_{r s}$, and the sinkage of the front idler $z_{f i}$, main straight part $z_{m p}$, and rear sprocket $z_{r s}$ and tangential force reveal different values due to the different amount of slippage at each of the grouser positions of the rigid link tracks at the bottom track elements of the front idler $i_{f}$, main straight parts $i_{m p}$, and rear sprocket $i_{r s}$ as shown in Fig. 2.

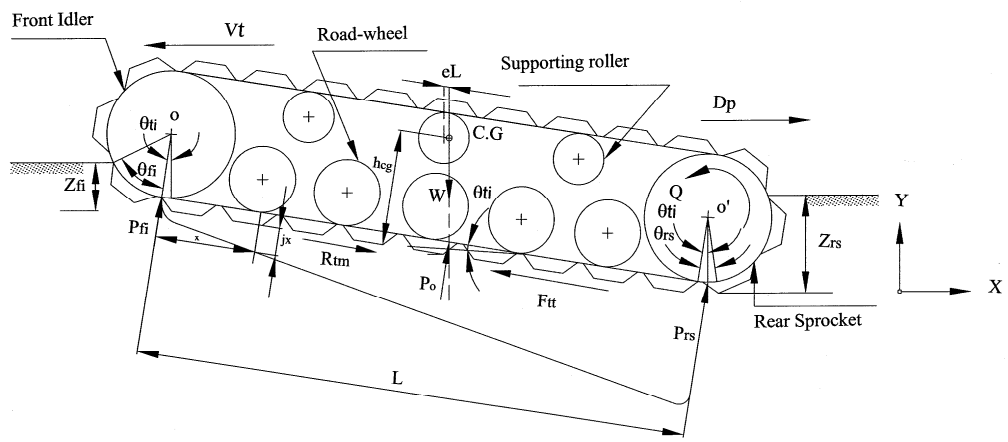

Fig. 2: Force acting on the track system of the vehicle during traversing on peat terrain with a slippage of $10 \%$. 
The following assumptions were made in order for the equation used in the mathematical modelling to be valided:

i. Vehicle theoretical speed was considered to be $10 \mathrm{~km} / \mathrm{hr}$ on zero slope terrain based on various off-road operations ASAE D497.3 NOV96, ASAE standard (1996).

ii. Vehicle total weight was considered to be $19.62 \mathrm{kN}$ with payload of $9.81 \mathrm{kN}$ based on the in-field maximum fresh bunches collection practiced.

iii. Vehicle's track critical sinkage was considered to be $0.1 \mathrm{~m}$ based on experimental data on Sepang Peat terrain [2].

iv. Aerodynamic resistance was neglected, due to the low operating speed.

v. Vehicle's belly drag was considered to be zero since the vehicle hull was not in contact with the terrain.

vi. Vehicle speed fluctuation was considered to be $2.75 \%$ based on Wong [7].

vii. Road-wheel spacing was considered to be $0.245 \mathrm{~m}$ to ensure good drawbar performance based on Wong [7].

\subsubsection{Slippage}

Slippage is one of the functional parameters for the vehicle traction mechanism. It reveals different value at the bottom track part of front idler, main straight part, and rear sprocket if the vehicle traverses on unprepared peat terrain with non-uniform ground pressure distribution. Therefore, it is important to compute the slippage of the front idler, track main straight part, and rear sprocket separately to determine the vehicle performance over the peat terrain.

For the slippage of the ground contact track of front idler, the relationship between the front idler slippage $i_{f i}$, the entry angle of the track at front idler $\theta_{f i}$, the track trim angle $\theta_{t i}$, the slippage ratio $i$ and front idler radius $R_{f i}$ can be modeled by the following equation [5].

$$
i_{f i}=\left(\frac{R_{f i}}{L_{f i}}\right)_{f i}\left[\theta_{f i}-(1-i)\left\{\sin \left(\theta_{f i}+\theta_{t i}\right)-\sin \theta_{t i}\right\}\right]
$$

where, the slip ratio, $i=\frac{v_{t}-v_{a}}{v_{t}}$, track trim angle, $\theta_{t i}=\arcsin \left(\left(z_{r s}-z_{f i}\right) / L\right)$, and track entry angle $\theta_{f i}=\arccos \left(\cos \theta_{t i}-z_{f i} / R_{f i}\right)$ and $L_{f i}=R_{f i}\left(\theta_{f i}+\theta_{t i}\right)$.

For the slippage of the ground contact track of rear sprocket, the relationship between the slippage of rear sprocket $i_{r s}$ and front idler $i_{f}$, rear sprocket radius $R_{r s}$, the track entry 
angle $\theta_{f i}$, the track trim angle $\theta_{t i}$, and exit angle $\theta_{r s}$ can be modeled by the following equation of Ataur et al.[5] :

$$
i_{r s}=i_{f i}+i\left(\frac{L_{r s}}{L}\right)+\left(\frac{R_{r s}}{L_{r s}}\right)\left[\theta_{r s}+(1-i) \sin \left(\theta_{r s}+\theta_{t i}\right)-\sin \theta_{t i}\right]
$$

where, track exit angle $\theta_{r s}=\arcsin \left(\frac{z_{r s}-z_{f i}}{L}\right)+\arccos \left[\frac{R_{r s}}{\sqrt{\left\{R_{r s}{ }^{2}-\left(z_{r s}^{2}-z_{f i}^{2}\right)\right\}^{2}}}\right]$, sinkage of the front idler, $z_{f i}=\frac{-\left(\frac{k_{p} D_{h f i}}{4 m_{m}}\right) \pm \sqrt{\left[\left(\frac{k_{p} D_{h f i}}{4 m_{m}}\right)^{2}+\frac{D_{h f i} p_{f i}}{m_{m}}\right]}}{2}$, sinkage of the rear sprocket, $z_{r s}=\frac{-\left(\frac{k_{p} D_{h r s}}{4 m_{m}}\right) \pm \sqrt{\left[\left(\frac{k_{p} D_{h r s}}{4 m_{m}}\right)^{2}+\frac{D_{h r s} p_{r s}}{m_{m}}\right]}}{2}$, hydraulic diameter of the terrain due to front idler, $D_{h f i}=\frac{4 B L_{f i b}}{2\left(L_{f i b}+B\right)}$, rear sprocket, $D_{h f i}=\frac{4 B L_{r s b}}{2\left(L_{r s b}+B\right)}$ and $L_{r s}=R_{r s}\left(\theta_{r s}+\theta_{t i}\right)$.

\subsubsection{Tractive Effort}

The tractive effort is denveloped not only on the ground contact part of the track but also on the side parts of the ground contact track grouser and on parts of front idler and rear sprocket. The initial track tension $12 \%$ of the total vehicle weight is assumed to be constant in every point of the track system in order to avoid the track deflection apparently between consecutive road-wheels.

\subsection{Ground Contact Part of the Track}

The traction mechanics of the track bottom part of the front idler, road-wheels, and rear sprocket are different due to its different angle of entry and exit. It is also different due to the different sinkage of the track front idler, main straight part, and rear sprocket when the vehicle traverses on the unprepared peat terrain with non-uniform ground pressure distribution. Therefore, it is important to compute the traction of the individual components bottom track segment, separately.

For the tractive effort developed at the track ground contact length of the front idler, the relationship between the tractive effort $F_{b}$ developed at the ground contact track, track ground contact length $L$, track width $B$, terrain cohesiveness $c$, normal stress $\sigma$, shear stress $\tau$, shear deformation modulus $K_{w}$, and slippage ratio of the track-terrain interfaces $i$ can be modeled by the following equation of Ataur et al.[5]:

$$
F_{f i b}=2 B L_{f i b}(c+\sigma \tan \varphi)\left[\frac{e^{1} K_{w f i}}{i_{f i} L_{f i b}}-\left(1+\frac{K_{w f i}}{i_{f i} L_{f i b}}\right) \exp \left(1-\frac{i_{f i} L_{f i b}}{K_{w f i}}\right)\right]
$$


with $L_{f i b}=R_{f i}\left(\theta_{f i}+\theta_{t i}\right)$

The tractive effort of the track main straight part and rear sprocket ground contact track was computed by Eq. (3).

\subsection{Side of the Ground Contact Track}

Wong [7] suggested that the traction mechanics of the track at the side of the grouser is highly significant on the development of vehicle traction if the vehicle sinkage is more than the grouser height. In this study, it was assumed that the sinkage of the vehicle was more than the grouser height of the vehicles track.

For the tractive effort developed at the side of the ground contact length of the front idler track, the relationship between the tractive effort $F_{s}$ developed at the side of the track ground contact part, track grouser height $H$, track ground contact length $L$, terrain cohesiveness $c$, normal stress $\sigma$, shear stress $\tau$, shear deformation modulus $K_{w}$, and slippage $i$ of the vehicle track-terrain interfaces can be modeled by the following equation of Ataur et al. [5]:

$$
F_{f i s}=4 H L_{f i b}(c+\sigma \tan \varphi) \cos \alpha\left[\frac{e^{1} K_{w f i}}{i_{f i} L_{f i b}}-\left(1+\frac{K_{w f i}}{i_{f i} L_{f i b}}\right) \exp \left(1-\frac{i_{f i} L_{f i b}}{K_{w f i}}\right)\right]
$$

$$
\text { with } \alpha=\arctan \left[\cot \left(\frac{H}{B}\right)\right]
$$

The tractive effort at the side of the track main straight part and rear sprocket ground contact track was computed using Eq. (4).

\subsection{Motion Resistance}

When the same vehicle as shown in Fig. 2, traverses on the peat terrain with nonuniform pressure distribution, the vehicle individual components such as front idler, main straight part, and rear sprocket reveal different values of motion resistances due to the different sinkages. Therefore, it is important to compute the motion resistance of the individual component for understanding the vehicle performance.

For the motion resistance of the vehicle due to terrain compaction $R_{c}$, the ground contact length $L$, track width $B$, sinkage of the vehicle $z$, stiffness of peat surface mat $m_{m}$ and underlying peat $k_{p}$ can be modeled by equation of Ataur et al. [5]:

$$
R_{c}=(2 B)\left[\begin{array}{l}
\left(\frac{L_{f i}}{L}\right)\left(\frac{k_{p} z_{f i}{ }^{2}}{2}+\frac{4}{3 D_{h f i}} m_{m} z_{f i}^{3}\right)+\left(\frac{L_{m p}}{L}\right)\left(\frac{k_{p} z_{m p}^{2}}{2}+\frac{4}{3 D_{h m p}} m_{m} z_{m p}{ }^{3}\right) \\
+\left(\frac{L_{r s}}{L}\right)\left(\frac{k_{p} z_{r s}{ }^{2}}{2}+\frac{4}{3 D_{h r s}} m_{m} z_{r s}{ }^{3}\right)
\end{array}\right]
$$


where

$$
D_{h f i}=\frac{4 B L_{f i}}{2\left(L_{f i}+B\right)}, D_{h m p}=\frac{4 B L_{m p}}{2\left(L_{m p}+B\right)}, \text { and } D_{h r s}=\frac{4 B L_{r s}}{2\left(L_{r s}+B\right)}
$$

For the motion resistance of the vehicle due to bull dozing effect, the relationship between the motion resistance of the vehicle due to bull dozing effect $R_{b}$, the bulk density of the terrain $\gamma_{\mathrm{d}}$, the internal frictional angle $\varphi$, track width $B$, and terrain cohesiveness $c$ can be modeled by the following general equation of Wong [7]:

$$
R_{b}=2 B\left[\gamma_{d} z^{2} \tan ^{2}\left(45+\frac{\varphi}{2}\right)+c z \tan \left(45+\frac{\varphi}{2}\right)\right]
$$

The total external motion resistance of the rubber track vehicle $R_{t m}$, can be computed as the sum of the individual motion resistance components by:

$$
R_{e t m}=R_{f i c}+R_{m s c}+R_{r s c}+R_{f i b}+R_{m s b}+R_{r s b}
$$

\subsection{Sprocket Torque}

When torque is applied at the sprocket, it starts driving the track and the vehicle starts moving. A frictional torque appears in the bearings of moving elements of the track system, resisting the vehicle motion. The forces appear at the track interface due to the terrain compaction and vehicle bulldozing effect, resisting the vehicle motion. Therefore, the vehicle needs to develop sufficient tractive effort after developing shear stress at the track-terrain interface in order to move forward and overcoming all of the motion resistance. The tension in each track segment does not affect the torque of the vehicle motion since it was assumed to be constant due to the geometrical arrangement of the road-wheel and initial tension equals to $12 \%$ of the total weight of the vehicle. For the torque of the sprocket, the relationship between the torque of the sprocket $Q$, vehicle total tractive effort $F_{t t}$, total motion resistance $R_{t m}$, sprocket radius $R_{r s}$, track grouser height $H$, vehicle total weight $W$, and vehicle normal reaction force $F_{n}$ and track ground contact length $L$ can be modeled by using the following equation:

$$
Q=D_{p}\left(R_{r s}+H\right)+W\left(h_{c g}-R_{r s}\right) \sin \theta_{t i}+L\left(F_{n}-W\right)(0.5-e) \cos \theta_{t i}
$$

where

$$
F_{n}=\frac{W-F_{t t} \sin \theta_{t i}}{\cos \theta_{t i}}
$$

\subsection{Vehicle Steerability}

The force system assumed to be acting on a tracked vehicle in general planar motion is shown in Fig. 3 and Fig. 4. In these figures, $F_{o t}$ and $F_{i t}$ are the thrusts on the outer and inner tracks. $R_{\text {lnot }}$ and $R_{\text {lnit }}$ are the longitudinal forces exerted by the terrain to per unit length of the outer and inner track, $f_{r}$ and $\mu_{l}$ are the coefficients of the longitudinal and 
lateral motion resistance, respectively, $B_{s t c}$ is the vehicle tracks tread, $F_{c e n t}$ is the centrifugal force and $\beta$ is the slip angle.

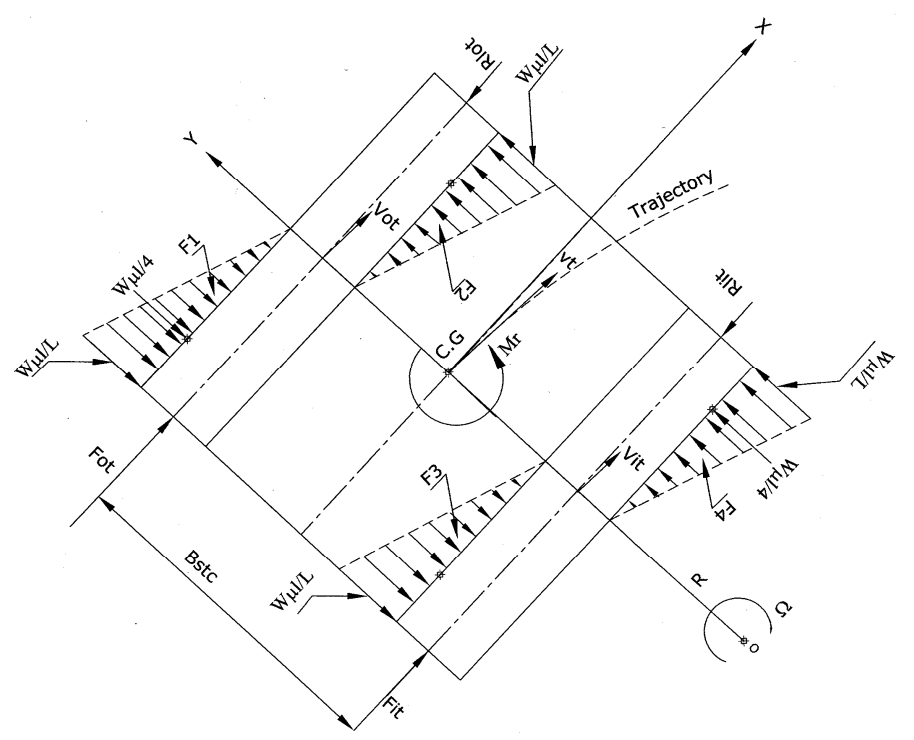

Fig. 3: Force acting on the track during turning at $6 \mathrm{~km} / \mathrm{hr}$.

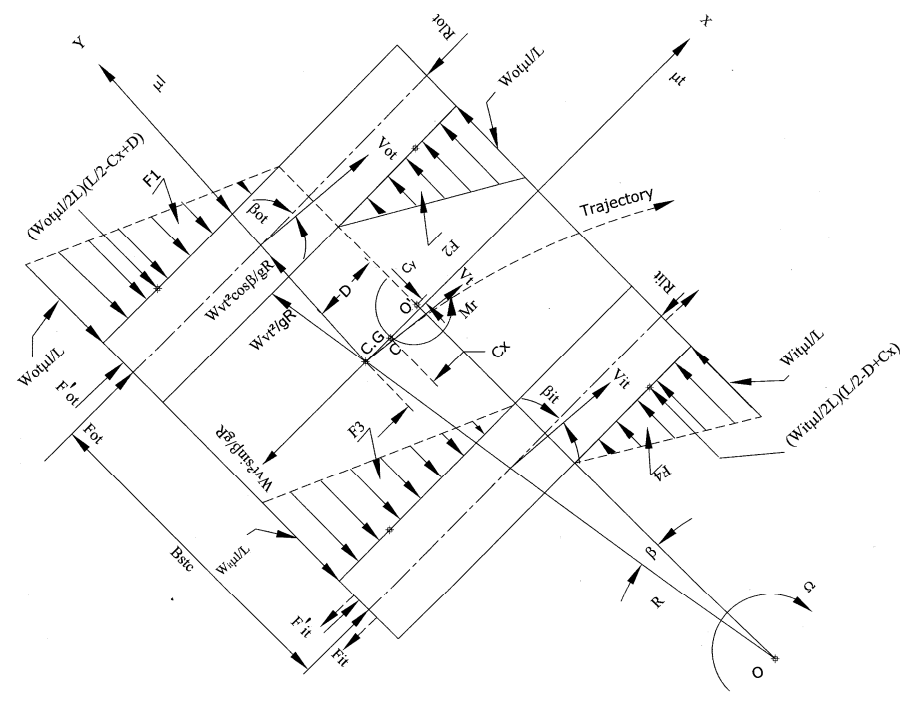

Fig. 4. Force acting on the track during turning at $10 \mathrm{~km} / \mathrm{hr}$.

During a turn, centrifugal force appears at the mass centre of the vehicle. When the vehicle turns at a traveling speed of $6 \mathrm{~km} / \mathrm{hr}$ with locating C.G at the middle of the track system, the slip angle of the vehicle is assumed to be zero due to negligible effect of centrifugal forces. The lateral motion resistance assumed to be equally distributed around the C.G of the vehicle and represented by equal triangles $F 1$ to $F 4$ as shown in Fig. 3. When the vehicle turns at a traveling speed of $10 \mathrm{~km} / \mathrm{hr}$ with locating C.G at $0.20 \mathrm{~m}$ rearward from the middle point of the track system, the slip angle of the track system $\beta$ is considered significant higher value and significantly affect the vehicle stability and 
steerability. Therefore, the lateral force distributions on the track system to be congruent are represented by the triangles F1 to F4 as shown in Fig. 4. The vehicle must have rotated about an instantaneous center point $\mathrm{O}^{\prime}$ located at a distance of $\mathrm{D}$ ahead of the vehicle C.G for the dynamic equilibrium. This force is generated by a shift to the point $\mathrm{O}^{\prime}$ ahead of $C . G$ and the vehicle will be oriented by an slip angle $\beta$, which can be computed as $\beta=\arctan \left(\Omega^{2} L / 4 g \mu_{l}\right)$, the yaw velocity $\Omega$ of the vehicle can be computed as $\Omega=\left(R_{r s}\right)\left(\frac{\omega_{o t}+\omega_{i t}}{2 R}\right)$,where, $R_{r s}$ is the radius of the sprocket. The vehicle follows the dashed trajectory, turning to the right around the instantaneous centre point $\mathrm{O}^{\prime}$ with turning radius $R$.

\subsection{Dynamic Load on the Tracks}

From the slippage equations of the outer and the inner track it can be identified that when the loading condition of the vehicle changes, as the vehicle is accelerating, the slippages of the vehicle track changes. Therefore, in order to adjust the vehicle slippage on given terrain it is necessary to estimate the proper loading condition for getting the high steerability of the vehicle The centrifugal force of the vehicle that will develop during turning at speed of $10 \mathrm{~km} / \mathrm{hr}$ can be computed as $F_{\text {cent }}=W \Omega^{2} R \cos \beta / g$, which causes lateral load transfer. The weight transfer from the inner track to the outer track is mainly due to the centrifugal force. If the centrifugal force is taken into consideration the dynamic loads of the outside and inside track will be different. For the dynamic load of the vehicle outer and inner track, the relationship between the dynamic load of outside and inside track $W_{o t}$ and $W_{i t}$, yaw movement of the vehicle $\Omega$, turning radius $R$, slip angle $\beta$ and acceleration due to gravity $g$, can be modeled using the following equations of Ataur et al.[3]:

$$
\begin{aligned}
& W_{o t}=\frac{W}{2}+\frac{h_{c g} W \Omega^{2} R}{B_{s t c} g} \cos \beta \\
& W_{i t}=\frac{W}{2}-\frac{h_{c g} W \Omega^{2} R}{B_{s t c} g} \cos \beta
\end{aligned}
$$

where $\beta=\arctan \left(\frac{D}{R}\right)$

\section{Drawbar pull}

The drawbar power is referred to as the potential productivity of the vehicle, that is, the rate at which productive work may be done. It is computed using the following equation:

$$
P_{d}=D_{p} V_{a}
$$

with $D_{p}=F_{t t}-R_{t m}$ 


\section{Tractive efficiency}

Tractive efficiency is used to characterize the efficiency of the track vehicle in transforming the engine power to the power available at the drawbar. It is defined by the following equation:

$$
\eta_{d}=\frac{P_{d}}{P_{e}}
$$

\section{MATHEMATICAL MODEL VALIDATION}

The drawbar pull and the tractive efficiency of light peat prototype Kubota Carrier RC20P track vehicle having track width of $0.43 \mathrm{~m}$, track ground contact length of $1.85 \mathrm{~m}$, total weight of $2645 \mathrm{~kg}$ including payload $1000 \mathrm{~kg}$ and three pneumatic road-wheels on each track, operating on a peat terrain were predicted through simulation. Basic parameters of the reference vehicle that used in the study are shown in Table 2.

Table 2. Basic parameters of Kubota Carrier RC20P track vehicle.

\begin{tabular}{lll}
\hline Parameters & Symbol & Values \\
\hline Vehicle parameters & & \\
$\quad$ Machine weight, kN & $\mathrm{W}$ & 1645 \\
$\quad$ Max. loading capacity, kN & $\mathrm{W}_{\mathrm{l}}$ & 1000 \\
$\quad$ Rated power, kW@ rpm & $\mathrm{P}$ & $8.2 @ 3200$ \\
& & \\
Track parameters & & \\
$\quad$ Ground contact length, m & $\mathrm{L}$ & 1.85 \\
Width, m & $\mathrm{B}$ & 0.43 \\
Grouser height, m & $\mathrm{H}$ & 0.05 \\
& & \\
Machine parameters & & \\
$\quad$ Length, m & $\mathrm{L}_{\mathrm{t}}$ & 2.98 \\
Width, m & $\mathrm{B}_{\mathrm{t}}$ & 1.76 \\
Height, m & $\mathrm{H}_{\mathrm{t}}$ & 1.45 \\
Ground clearance, $\mathrm{m}$ & $\mathrm{G}_{\mathrm{c}}$ & 0.27 \\
\hline
\end{tabular}

Source: Ooi [6]

Table 3 shows that the regression model is highly significant at a significance level of $\mathrm{P}_{\mathrm{r}}<0.01$. In the regression model, $\mathrm{t}$ values of 11.74 for $i$ and -7.84 for $i^{2}$ are higher than $t * 16(0.01)=2.69$. Hence, the null hypothesis is rejected. This implies that the slippage of the vehicle has significant effect on the drawbar pull of the vehicle. A standard error value of 0.032 for $i$ and 0.00067 for $i^{2}$ are concluded that both of the predicted and measured drawbar pull is pretty tightly bunched together. The variability of the predicted and measured drawbar pulls of the vehicle has the less variability around the best fitted regression line, $D_{p}=0.916+0.385(i)-0.005\left(i^{2}\right)$ where, $D_{p}$ is the drawbar pull of the vehicle in $\mathrm{kN}$ and $i$ is the slippage of the vehicle in percentage. Therefore, based on the 
vehicle estimated standard error of 0.032 and the R-square values of 0.967 , it can be concluded that the predicted and the measured drawbar pull are strongly correlated.

Table 3: Regression analysis on the drawbar pull of Kubota Carrier RC20P track vehicle.

\begin{tabular}{cccccc}
\hline Source & df & SS & F value & P value & R square \\
\hline Model & 2 & 99.97650 & 49.98825 & 0.0001 & 0.9667 \\
Error & 16 & 4.52226 & 0.28264 & \\
C total & 18 & 104.49876 & & \\
& & & \\
Estimated & Coefficient & T for H0: & Standard Error & $\mathrm{P}_{\mathrm{r}}<[\mathrm{T}]$ \\
Parameter & & Parameter $=0$ & & 0.0034 \\
Intercept & 0.916190 & 3.431 & 0.26699892 & 0.0001 \\
$i$ & 0.385447 & 11.794 & 0.03268138 & 0.0001 \\
$i^{2}$ & -0.005228 & -7.842 & 0.00066673 & \\
\hline
\end{tabular}

Table 4 shows that the slippage of the vehicle significantly $\left(\mathrm{P}_{\mathrm{r}}<0.01\right)$ affects the vehicle drawbar pull. While, the non-significant value of treatments (predicted and measured drawbar pull) indicates that there is no significant difference between the treatments. Therefore, the close agreement between the predicted and measured drawbar pull of the vehicle substantiates the validity of the simulation model.

Table 4: ANOVA on the drawbar pull of Kubota Carrier RC20P track vehicle

\begin{tabular}{lllll}
\hline Slippage & 18 & 201.47489383 & 143.21 & 0.0001 \\
\hline Treatment & 1 & 0.14455325 & 1.85 & 0.1906 \\
Error & 18 & 1.40688027 & & \\
Total & 37 & 203.02632735 & & \\
\hline
\end{tabular}

Table 5 shows that the regression model is highly significant at significance level of $\mathrm{P}_{\mathrm{r}}<0.01$. In the regression model, $\mathrm{t}$ values of 2.91 for $i$ and -3.81 for $i^{2}$ are higher than $t^{*}{ }_{35}(0.01)=2.67$. Hence, the null hypothesis is rejected. This implies that the slippage of the vehicle has significant effect on the tractive efficiency of the vehicle. The standard error values of 0.23 for $i$ and 0.0087 for $i^{2}$ concluded that both of the predicted and measured drawbar pull are pretty tightly bunched together. The variability of the predicted and measured drawbar pulls of the vehicle have less variability around the best fitted regression line, $T=56.61+1.25(i)-0.0034\left(i^{2}\right)$ where, $\mathrm{T}$ is the tractive efficiency of the vehicle in percentage and $i$ is the slippage of the vehicle in percentage. Therefore, based on the standard error estimated values of the vehicle of 0.23 and the R-square value of 0.79 , it can be concluded that the predicted and the measured drawbar pulls are strongly correlated. 
Table 5: Regression analysis on the tractive efficiency of Kubota Carrier RC20P track vehicle.

\begin{tabular}{llllll}
\hline Source & df & SS & F value & P & \\
\hline Model & 2 & 2604.69 & 13.31 & 0.000 & 0.79 \\
Error & 35 & 3426.12 & & & \\
C total & 37 & 6030 & & & \\
Estimate & Coefficien & T for H0: & Standar & $\mathrm{P}_{\mathrm{r}}<[\mathrm{T}]$ \\
Intercept & 56.61 & 15.83 & 3.51 & 0.0001 \\
\hline
\end{tabular}

Table 6 shows that the slippage of the vehicle significantly $\left(\mathrm{P}_{\mathrm{r}}<0.01\right)$ affects the tractive efficiency of the vehicle. The non-significant value of treatments (predicted and measured tractive efficiency) indicates that there is no significant difference between the treatments on the model. Therefore, the closed agreement between the predicted and measured tractive efficiency of the vehicle substantiates the validity of the simulation model.

Table 6: ANOVA on the tractive efficiency of Kubota Carrier RC20P track vehicle.

\begin{tabular}{lllll}
\hline Source & \multicolumn{1}{c}{ df } & \multicolumn{1}{c}{ SS } & F value & P value \\
\hline Slippage & 18 & 6153.59 & 2556.23 & 0.0001 \\
Treatment & 1 & 0.006 & 0.05 & 0.83 \\
Error & 18 & 2.28 & & \\
Total & 37 & 203.026 & & \\
\hline
\end{tabular}

\section{VEHICLE DESIGN PARAMETERS OPTIMIZATION}

Tractive performance of the rigid link segmented rubber tracked vehicle has been computed with the computer simulation method based on the new mathematical model for undrained peat terrain. It appeared that the engine size and tractive performance of the vehicle on peat terrain vary with: the variation of vehicle weight, track size including track ground contact length, width, pitch and grouser height track entry and exit angle, idler diameter and location, sprocket diameter and location, road-wheel diameter, spacing and geometrical arrangement, and location of center of gravity. Therefore, for the selection and optimization design parameters of the vehicle track size, idler diameter and location, sprocket diameter and location, number of road-wheel, road-wheel diameter, spacing and geometrical arrangement, ratio of the road wheel spacing to track pitch, ratio of the sprocket diameter to track pitch, and the location of the center of gravity are taken into account. The optimization design parameters of the vehicle have been performed by using the Microsoft Excel software with performing calculations, analyzing information and managing lists in spreadsheets. 


\subsection{Track Width and Ground Contact Length}

The length of the track in contact with the ground and the level of pressure within the ground are the most important factors that influenced tracked vehicle tractive performance. To evaluate the effects of track system configuration on the vehicle ground pressure distribution and surface mat stiffness, it is important to study track ground contact length and width. Figures 5(a) and 5(b) show that the vehicle ground pressure distribution decreases with increasing vehicle track ground contact length and width. The vehicles under consideration are traversing on a zero slope terrain with a travel speed of $10 \mathrm{~km} / \mathrm{hr}$. From the field experiment on Sepang, it was found that the bearing capacity for the undrained peat terrain was $17 \mathrm{kN} / \mathrm{m}^{2}$. It appears that if a ground contact pressure of the $19.62 \mathrm{kN}$ vehicle, with a moderate payload of $5.89 \mathrm{kN}$, is limited to $16.35 \mathrm{kN} / \mathrm{m}^{2}$ by designing a track with ground contact area of $30 \times 2000 \mathrm{~mm}^{2}$ then the sinkage and external motion resistance of the vehicle will be low and tractive effort will be high, yielding a desired travel speed of $10 \mathrm{~km} / \mathrm{hr}$ and vehicle productivity.
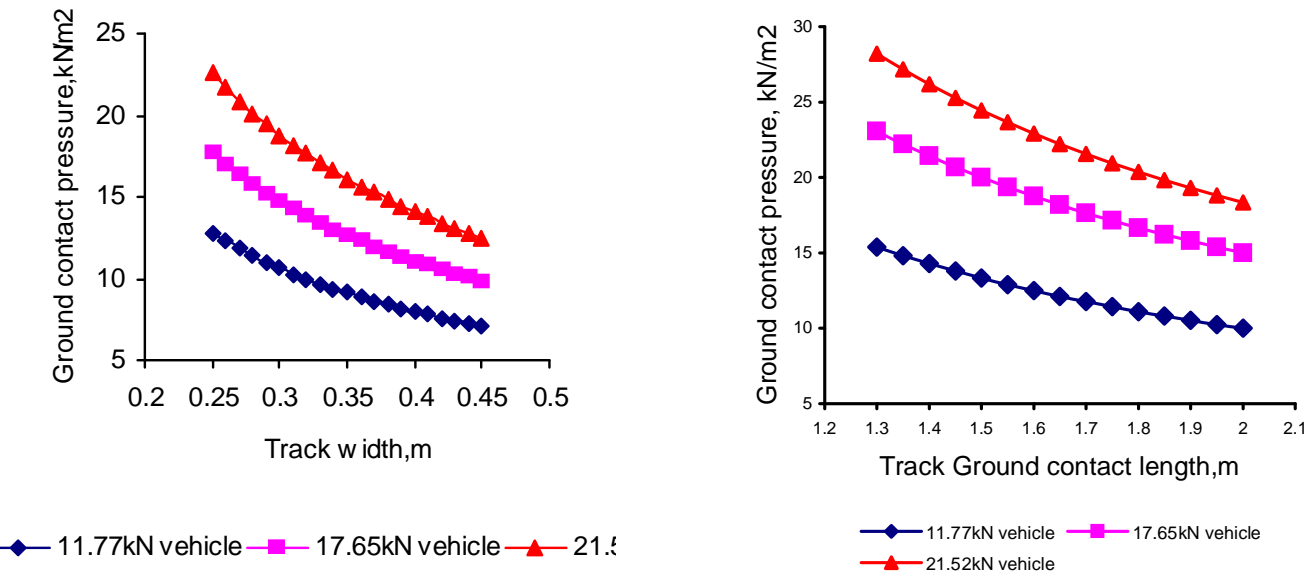

Fig. 5: Variation of ground pressure distribution with (a) variation of track width at constant track ground contact length of $200 \mathrm{~cm}$ and (b) variation of track ground contact length at constant track width of $30 \mathrm{~cm}$.

Figures. 6 (a) and 6(b) show that the sinkage of the vehicle decreases with increasing track width and track ground contact length. If the track size of the vehicles is limited to $300 \times 2000 \mathrm{~mm}^{2}$, then the sinkage of the $11.77 \mathrm{kN}, 17.65 \mathrm{kN}$, and $21.52 \mathrm{kN}$ vehicles will be $61,81.8$, and $110 \mathrm{~mm}$, respectively. From the field experiment, it was found that the surface mat thickness of the Sepang peat terrain was $100 \mathrm{~mm}$, which will support the maximum load of the vehicle during static and dynamic as well. Therefore, if the vehicle sinkage is more than $100 \mathrm{~mm}$ the vehicle will sink rather than traverse. If the vehicle total weight is considered to $19.62 \mathrm{kN}$ and the track ground contact area to $300 \times 2000 \mathrm{~mm}^{2}$, the vehicle will traverse on the peat terrain with sinkage of $90 \mathrm{~mm}$ or $10 \%$ less than the Sepang peat terrain surface mat thickness and exit ground pressure of $16 \mathrm{kN} / \mathrm{m}^{2}$ or $6 \%$ less than the worst condition Sepang peat terrain bearing capacity. Based on the $19.62 \mathrm{kN}$ vehicle sinkage and ground contact pressure, it may be conclude that the vehicle will not 
in risk to traverse on peat terrain if the vehicle used the track ground contact area of $300 \times 2000$ $\mathrm{mm}^{2}$.
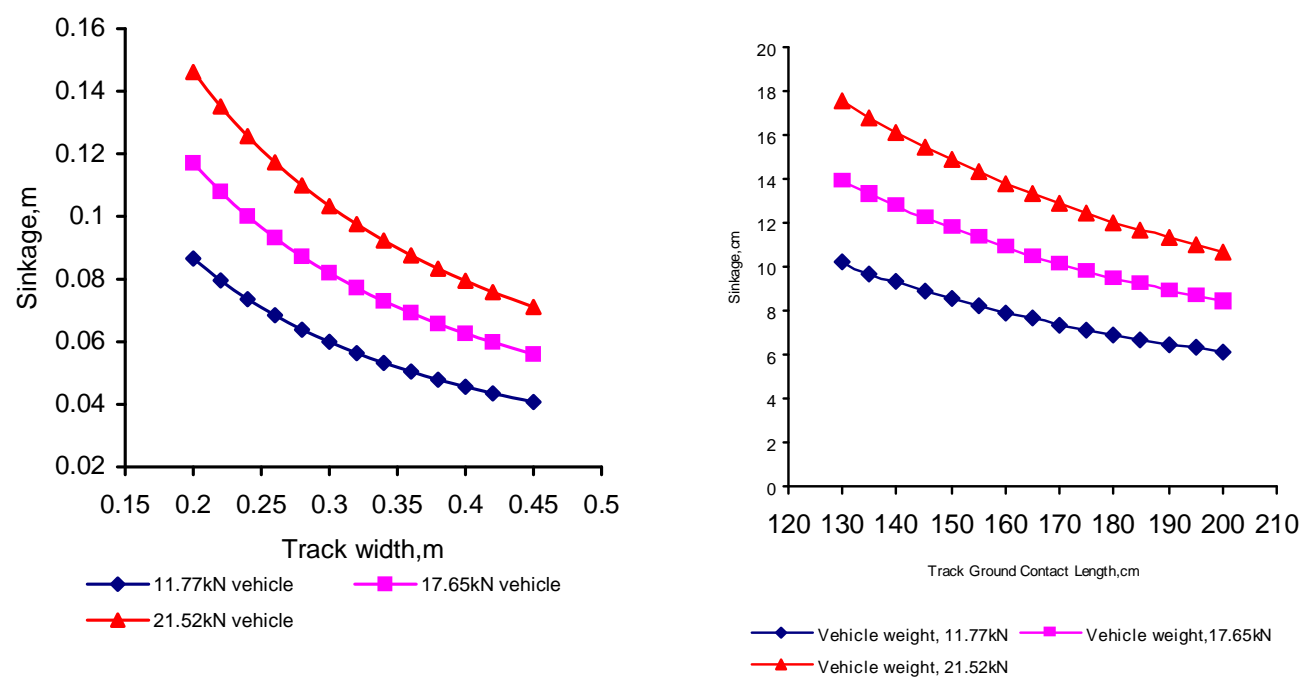

Fig. 6: Variation of vehicle sinkage with (a) variation of track width at constant track ground contact length of $200 \mathrm{~cm}$ and (b) variation of track ground contact length at constant track width of $30 \mathrm{~cm}$.

Therefore, the best choice is to select a vehicle track ground contact area of 300x2000 $\mathrm{mm}^{2}$ for the vehicle to produce an effective tractive performance.

The conclusion is further supported by the relation between the track size and motion resistance with keeping option either track width or track ground contact length could increase to adjust the track ground contact area for getting the desired vehicle ground contact pressure. Figures 7(a) and 7(b) show that the motion resistance coefficient of the vehicle increases with increasing track width and decreases with increasing track ground contact length. Figure 7(a) shows that the motion resistance coefficient increased $18.12 \%$ for $13.73 \mathrm{kN}$ vehicle, $16.99 \%$ for $17.65 \mathrm{kN}$ vehicle and $24.12 \%$ for $21.58 \mathrm{kN}$ vehicle with increasing the track width from 0.2 to $0.4 \mathrm{~m}$ when the track ground contact length considered to keep in constant at $2.0 \mathrm{~m}$. Whereas, Fig. 7(b) shows that the vehicle motion resistance coefficient of the vehicle decreased $21.09 \%$ for $13.73 \mathrm{kN}$ vehicle, $24.07 \%$ for $17.65 \mathrm{kN}$ vehicle and $24.5 \%$ for $21.58 \mathrm{kN}$ vehicle with increasing the track ground contact length from 1.3 to $2.2 \mathrm{~m}$ when the track width considered to keep in constant at $0.3 \mathrm{~m}$. From the justification of vehicle motion resistance coefficient based on vehicle track width and track ground contact length, it could be noted that the vehicle track ground contact length should be considered to increase instead of increase the track width in order to get the vehicle lower ground contact pressure of $16 \mathrm{kN} / \mathrm{m}^{2}$ on Sepang peat terrain. Therefore, it was found that for a given overall dimension of $300 \times 2000 \mathrm{~mm}^{2}$ track system, the maximum motion resistance coefficient of the $19.62 \mathrm{kN}$ vehicle is $5.4 \%$, which could be good enough for a track vehicle on soft terrain [7]. 

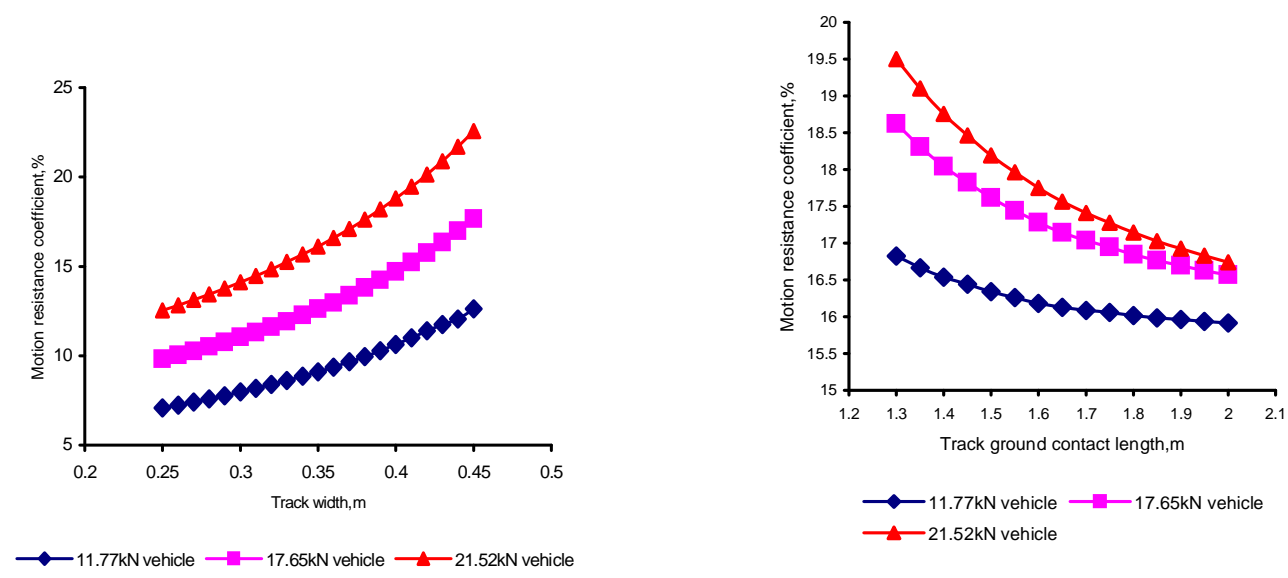

Fig. 7: Effect of track size on vehicle tractive performance (a) track width and (b) track ground contact

Based on Figs. 8(a) and 8(b), it could be pointed out that if the $19.62 \mathrm{kN}$ vehicle track size is considered to be $300 \times 2000 \mathrm{~mm}^{2}$, the vehicle ground pressure exit on track-terrain interfaces is $16 \mathrm{kN} / \mathrm{m}^{2}$ with sinkage of $90 \mathrm{~mm}$ and motion resistance coefficient of $5.4 \%$. Therefore, the $19.62 \mathrm{kN}$ vehicle track system overall dimension can be optimized by selecting track width of $300 \mathrm{~mm}$ and ground contact length of $2000 \mathrm{~mm}$.

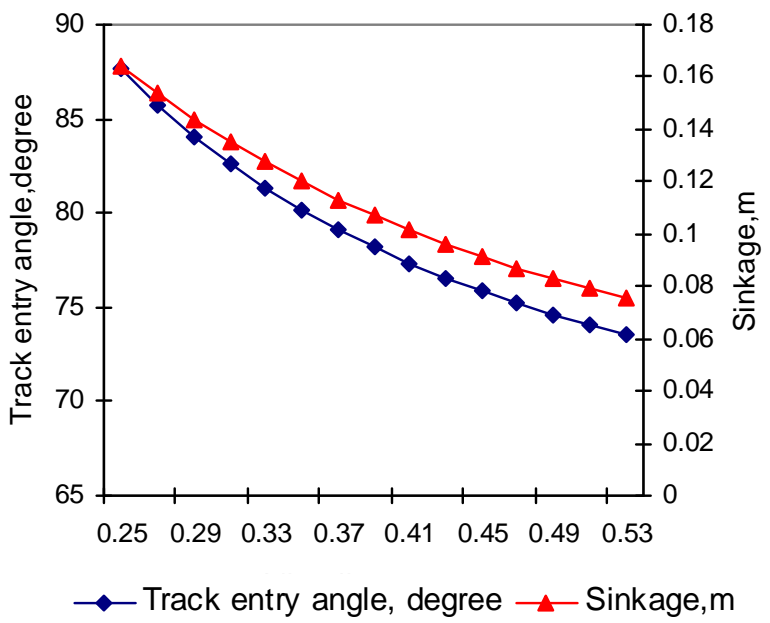

Fig. 8: Relationship between track entry angle, sinkage and idler diameter.

\subsection{Track Grouser Size}

To fully utilize the shear strength of the peat surface mat for generating tractive effort, the use of grouser on tracks would be required. From the field experiment on Sepang, it was found that the shear strength of the peat surface mat is considerably higher than that of the underlying peat deposit and that there is well defined shear-off point beyond which the resistance to shearing is significantly reduced. This would, however, considerably 
increase the risk of tearing off the surface mat unless the slip of the track is properly controlled. Thus, the use of aggressive grouser on vehicles for use in organic terrain does not appear to be desirable from traction as well as environmental viewpoints. The surface mat thickness of Sepang peat terrain was found to be about $0.1 \mathrm{~m}$. In order to fully utilize the shear strength of the surface mat and to increase the traffic ability of the terrain the grouser height of the track is considered to be $0.06 \mathrm{~m}$.

\subsection{Sprocket Location and Size}

The location of drive sprocket has a noticeable effect on the vehicle tractive performance. Wong et al. [6] (1986) reported that in forward motion, the top run of the track is subjected to higher tension when the sprocket is located at the front than when the sprocket is located at the rear. Thus, with a front sprocket drive, a larger proportion of the track is subjected to higher tension and the overall elongation and internal losses of the track will be higher than with a rear sprocket drive. With higher elongation, more track length is available for deflection and the track segments between road-wheels take fewer loads and the vibration of the track increase, which will cause the fluctuation of the track. Consequently, the sinkage and motion resistance will be higher and the mobility of the vehicle will be affected severely on the unprepared peat terrain. Therefore, the sprocket could be considered to locate at the rear part of the track system configuration in order to distribute the vehicle normal pressure to the track-terrain interfaces uniformly. The center point of the sprocket is considered the $(0,0)$ coordinate system of the vehicle.

Generally, it could be mentioned that the sprocket is the most important component of the vehicle track system, which propels the vehicle with sufficient torque, control the vehicle speed fluctuation and maintain the vehicle tractive performance. Therefore, the size of the sprocket can be determined from the relationship between the relationship between the sprocket torque, vehicle speed fluctuation, and vehicle turning radius. From the simulation result, it was found that the ratio of the sprocket diameter to track pitch have significant effect on the vehicle tractive performance. Therefore, the ratio of the sprocket pitch diameter to track pitch should be a value which will stand to meet the field requirement.

Further support to optimize the sprocket size of the vehicle track system, the following equation on vehicle speed fluctuation can be considered. For the relation of vehicle speed fluctuation and the ratio of the vehicle sprocket pitch diameter to tract pitch the following mathematical model of Wong [7]can be used:

$$
\delta=1-\sqrt{\left[1-\left(\frac{1}{D_{p r s} / T_{p}}\right)^{2}\right]}
$$

where $\delta$ is the speed fluctuation in percentage, $D_{p r s} / T_{p}$ is the sprocket pitch diameter to track pitch in proportion.

Using $D_{\text {prs }} / T_{p}$ equals to 4.00 , the computed value of $\delta$ is $3.17 \%$. According to Wong [7], the industrial and agricultural track vehicle speed fluctuation should be in the range of 3.72 to $2.75 \%$. Since the speed fluctuation of the vehicle was found of $3.17 \%$, the ratio of 
the vehicle sprocket pitch diameter to track pitch can be optimized at 4.00. Consequently, the sprocket pitch diameter was optimized at $400 \mathrm{~mm}$ by using the track pitch of $100 \mathrm{~mm}$.

\subsection{Idler location and size}

Idler is located at $-2.0 \mathrm{~m}$ front of the track system. It was earlier reported that the surface mat thickness of Sepang peat terrain in the ranged of 100 to $250 \mathrm{~mm}$ which is considered the supporting platform of the vehicle. It could be noted that if the sinkage of any vehicle on the Sepang peat terrain is more than $100 \mathrm{~mm}$ will cause the vehicle to bog down. Furthermore, from the simulation it was found that the track entry angle was significantly affect the vehicle front idler size and tractive performance. Therefore, from the relationship between the vehicle sinkage, track entry angle and idler diameter, the idler diameter can be identified. Figure 8 shows that the vehicle track entry angle at front idler and sinkage decreases with increasing vehicle front idler diameter. If the vehicle critical sinkage of the vehicle is considered to $100 \mathrm{~mm}$, the corresponding front idler diameter and track entry angle were found $400 \mathrm{~mm}$ and $78^{\circ}$, respectively.

This conclusion can be further supported from the relationship between the track entry angle, slippage and vehicle tractive performance. Figure 9 shows that the relationship between the vehicle track entry angle, slippage, and tractive efficiency. At track entry angle $78^{\circ}$, the vehicle slippage and tractive efficiency were found $18 \%$ and $70.5 \%$, respectively, which was found at sprocket pitch diameter of $400 \mathrm{~mm}$. Therefore, the front idler diameter $400 \mathrm{~mm}$ can be optimized at $400 \mathrm{~mm}$ for getting the tractive efficiency of the vehicle $70.5 \%$ and high productivity.

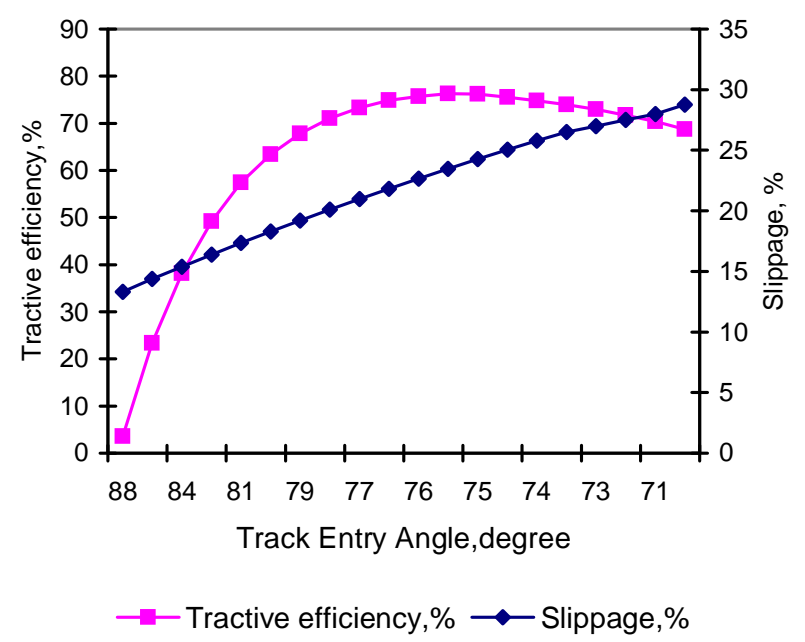

Fig. 9: Track entry angle, tractive performance and slippage.

\subsection{Roadwheel diameter, Track pitch, and Number of Roadwheel}

Wong [7] reported that the ratio of road wheel spacing to track pitch is a significant parameter that affects the tractive performance of tracked vehicle, particularly on soft terrain. The decrease in the track motion resistance coefficient with the increase of the number of road wheels was primarily due to the reduction in the peak pressures and 
sinkage under the road wheels. The longer track pitch would lead to an improvement in tractive performance over soft terrain. But, it may cause a wider fluctuation in vehicle speed and higher associated vibration.

Consequently, a proper compromise between tractive performance and smoothness of operation must be struck. Road-wheel diameter can be predicted based on the following equation:

$$
S_{r}=\frac{D_{1}}{2}+\frac{D_{2}}{2}+G
$$

where, $S_{r}$ is the road-wheel spacing in $\mathrm{mm}, D_{1}$ is the first road-wheel diameter in $\mathrm{mm}, D_{2}$ is the second road-wheel diameter in $\mathrm{mm}$ and $G$ is the gap between consecutive roadwheel is assumed to be $5 \mathrm{~mm}$ for avoiding the track deflection between the consecutive road-wheel.

In the track system all the road-wheel dimension $\left(D_{1}=D_{2}=-----=D_{7}\right)$ are considered as equal size. If the roadwheel spacing equals to $225 \mathrm{~mm}$, the gap between two consecutive road-wheel on the track system equals to $5 \mathrm{~mm}$, the computed value of roadwheel diameter equals to $220 \mathrm{~mm}$.

Figure 10 shows that the vehicle drawbar pull increases with increasing the ratio roadwheel spacing to track pitch and tractive efficiency increases with increasing the ratio of road-wheel spacing to track pitch until 2.1 and then decreases with further increasing of the ratio of road-wheel spacing to track pitch. If the ratio of road-wheel spacing to track pitch is considered to be 2.25 , the tractive efficiency of the vehicle is found $70.5 \%$. Whereas, the tractive efficiency of the vehicle is found $70.5 \%$ for the optimum sprocket pitch diameter of $400 \mathrm{~mm}$ and idler diameters of $400 \mathrm{~mm}$. Therefore, the ratio of roadwheel spacing to track pitch should be 2.25 if the optimum sprocket pitch diameter and idler diameters each is limited to $400 \mathrm{~mm}$. By using $S_{r} / T_{p}$ equals to 2.25 and $S_{\mathrm{r}}$ equals to $225 \mathrm{~mm}$, the computed value of $T_{p}$ equals to $100 \mathrm{~mm}$.

The number of road-wheels can be computed based on Fig. 2 by the following equation [5]:

$$
n_{r}=\frac{\left(L-\left(\left(D_{r s}+D_{f i}\right) / 2\right)\right)}{\left(D_{r}+G\right)}
$$

where, $L$ is the total ground contact length in $\mathrm{mm}, D_{r s}$ is the outside diameter of the sprocket in $\mathrm{mm}, D_{f i}$ and $D_{r}$ are the diameter of the front idler and road-wheel in mm and $n_{r}$ is the number of road-wheel. The outside diameter of the sprocket (i.e, $D_{r s}=D_{p r s}+H / 2$ ) is considered to $460 \mathrm{~mm}$ based on the grouser height.

By using $L$ equals to $200 \mathrm{~mm}, D_{r s}$ equals to $460 \mathrm{~mm}, D_{f i}$ equals to $400 \mathrm{~mm}, D_{r}$ equals to $220 \mathrm{~mm}, G$ equals to $5 \mathrm{~mm}$, the computed value of $n_{r}$ is 7 . Therefore, total number of roadwheel seven with diameter of $220 \mathrm{~mm}$ on the $19.62 \mathrm{kN}$ vehicle track system would significantly reduce vehicle vibration during traversing on the unprepared peat terrain by making zero deflection of the track between two consecutive road-wheel. 


\subsection{Center of Gravity Location}

Center of gravity of a tracked vehicle is a most important design parameter for getting the high tractive performance. Figure 10 shows that the tractive efficiency of the vehicle increases steeply with increasing the slippage of the vehicle until a certain value and then start to decrease with increasing the slippage of the vehicle. The vehicle under consideration with total weight $19.62 \mathrm{kN}$ including payload of $5.89 \mathrm{kN}$ is traversing on a zero slope terrain with traveling speed of $10 \mathrm{~km} / \mathrm{hr}$. Figure 11 shows the maximum tractive efficiency of $79.8 \%$ at $11 \%$ slippage for the vehicle with center of gravity located at 300 $\mathrm{mm}$ rearward from the mid-point of the track ground contact length and $70.5 \%$ at $12 \%$ slippage for the vehicle with center of gravity located at the mid-point of the track ground contact length.

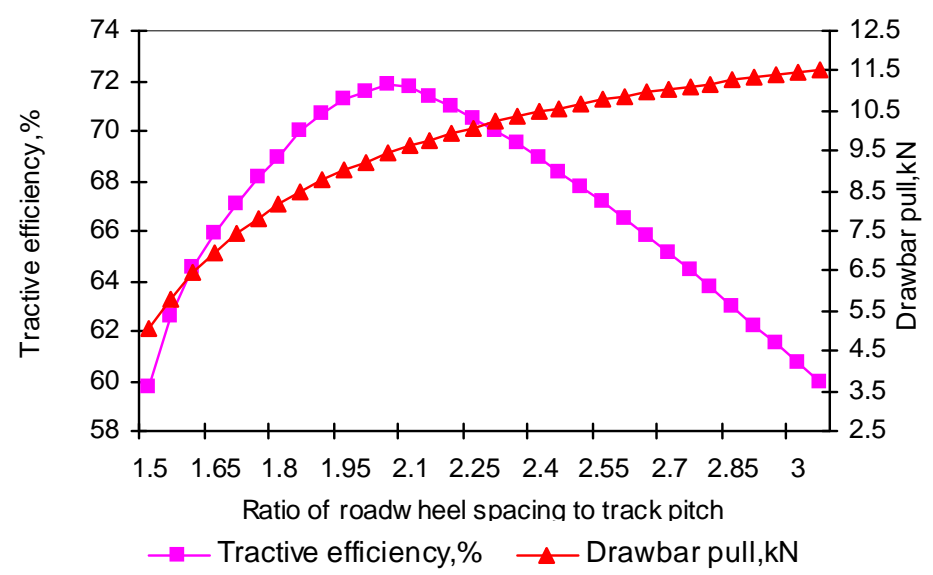

Fig. 10: Relationship between tractive efficiency, drawbar pull and the ratio of the road-wheel spacing to track pitch

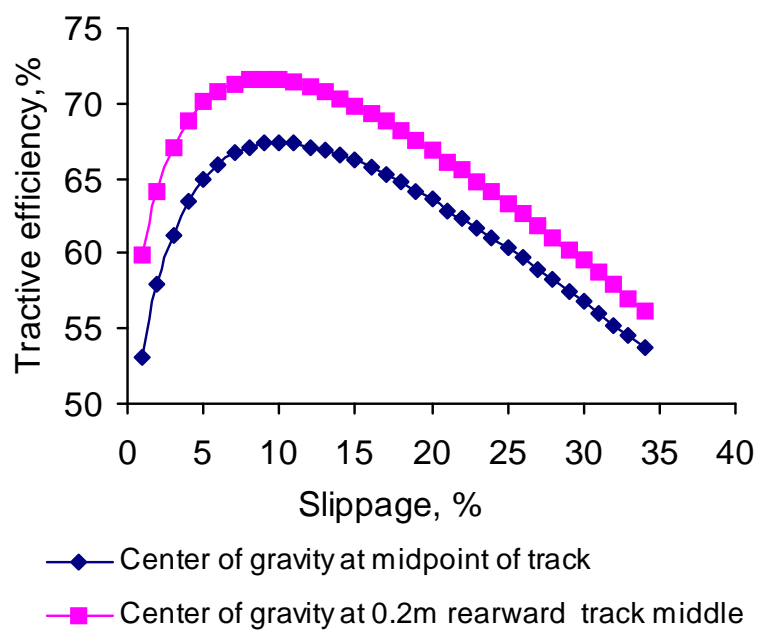

Fig. 11: Relationship between tractive efficiency and slippage. 
From the comparison of the vehicle based on the location of center of gravity, it is found that the tractive efficiency of the vehicle with center of gravity is located at $200 \mathrm{~mm}$ rearward from the mid point of the track ground contact length is $13.2 \%$ higher than the tractive efficiency of the vehicle with the center of gravity is located at the mid-point of the track ground contact length. The variation of tractive efficiency is found between the vehicle with the locations of center of gravity due to the difference of external motion resistance. It could be pointed out that the vehicle with location of center of gravity at $200 \mathrm{~mm}$ rearward from the mid point of the track ground contact length reveals lower sinkage at the frontal part of the track ground contact part causes the lower external motion resistance and the vehicle consume lower engine power for developing effective tractive effort in order to traverse the vehicle easily on the low bearing capacity peat terrain. Whereas, the vehicle with location of center of gravity at the midpoint of the track ground contact length reveals the equal sinkage to all over the ground contact part causes the higher external motion resistance and vehicle consume maximum engine power for developing the required tractive effort in order to traverse the vehicle on the low bearing capacity peat terrain. Therefore, the vehicle center of gravity location of $300 \mathrm{~mm}$ rearward from the mid-point of the track ground contact length could be optimized the center of gravity location for the vehicle. The basic design parameters of the vehicle found from the simulation study are shown in Table 7

Table 7. Basic design parameters of the special segmented rubber tracked vehicle

\begin{tabular}{lll}
\hline Vehicle Parameters & & \\
\hline Total weight including $9.81 \mathrm{kN}$ payload, $\mathrm{kN}$ & $v_{t}$ & 19.62 \\
Vehicle traveling speed, $\mathrm{km} / \mathrm{hr}$ & $x_{c g}$ & -0.80 \\
Center of gravity, x coordinate, m & $y_{c g}$ & 0.45 \\
Centre of gravity, y coordinate, m & $D_{r s}$ & 0.40 \\
Sprocket pitch diameter, m & $D_{f i}$ & 0.40 \\
Idler diameter, m & $x_{c f i}$ & -2.0 \\
Idler center, x coordinate, m & $y_{c f i}$ & 0 \\
Idler center, y coordinate, m & $n$ & 7 \\
Number of road-wheels (each side) & $D_{r}$ \\
Road-wheel diameter, m & $S_{r}$ & 0.22 \\
Road-wheel spacing, m & $n_{s}$ & 0.225 \\
Number of supporting rollers (each side) & $D_{s}$ \\
Supporting rollers diameter, m & & 0.20 \\
Track Parameters & $L_{c}$ & 5.90 \\
Track total length (each side), m & $T_{p}$ & 0.10 \\
Track pitch, m & $\mathrm{B}$ & 0.30 \\
Track width, m & $L$ & 2.00 \\
Track ground contact length, m & $S_{r} / T_{p}$ & 2.25 \\
Road-wheel spacing to track pitch & $\delta$ & 3.17 \\
Vehicle speed fluctuation, percentage & $H$ & 0.06 \\
Grouser height, m & $H$ & \\
\hline
\end{tabular}

Note: Coordinates origin is at the center of the sprocket. Positive $\mathrm{x}$ and $\mathrm{y}$ coordinates are to the rear and top, respectively. 


\section{CONCLUSION}

The following conclusions were made based on the analysis of this paper:

Based on the results of mechanical properties of peat in the area studied, the mean values for moisture content of $79.58 \%$, bulk density of $1.53 \mathrm{kN} / \mathrm{m}^{3}$, cohesiveness of $1.36 \mathrm{kN} / \mathrm{m}^{3}$, internal friction angle of $26.22^{\circ}$, shear deformation modulus of $11.2 \mathrm{~mm}$, surface mat stiffness of $13.6 \mathrm{kN} / \mathrm{m}^{3}$, and underlying peat stiffness of $171.54 \mathrm{kN} / \mathrm{m}^{3}$.

Based on the results of detailed study on vehicle parameters, an optimized track system configuration for the $19.62 \mathrm{kN}$ vehicle has seven roadwheels with diameter of $0.24 \mathrm{~m}$, a track pitch of $0.1 \mathrm{~m}$, a ratio of the initial track tension to vehicle weight of $12 \%$, a location of center of gravity at $30 \mathrm{~cm}$ rearward of the mid-point of the track ground contact length ensure the vehicle to develop the maximum tractive efficiency of $74 \%$ during traversing at $10 \mathrm{~km} / \mathrm{hr}$ on the specified peat terrain.

Based on the simulation study, it was found that the track pitch, number of road wheels, and location of centre of gravity have noticeable effects on the tractive performance of the vehicle. The maximum tractive efficiency of the vehicle is in the range of 74 to $72 \%$ and 50 to $48 \%$ with the slip range of 9 to $12 \%$ when the vehicle traveled at $10 \mathrm{~km} / \mathrm{hr}$ without payload and with payload, respectively. Furthermore, the tractive efficiency of the vehicle with center of gravity located at $300 \mathrm{~mm}$ rearward of the mid-point of track ground contact length are $10 \%$ higher than the vehicle center of gravity located at the mid-point of track ground contact length.

\section{ACKNOWLEDGEMENT}

This research project is classified under RM7 IRPA Project No. 01-02-04-0135. The authors are very grateful to the Ministry of Science, Technology and the Environment of Malaysia for granting the financial assistance.

\section{REFERENCES}

[1] ASAE, Agricultural Engineers Yearbook of Standards, American Society of Agricultural Engineers, Michigan,1996.

[2] R. Ataur, Y. Azmi, M. Zohadie, A. Desa, W. Ishak, and A. Kheiralla, " Mechanical Properties in Relation to Vehicle Mobility of Sepang Peat Terrain in Malaysia", Journal of Terramechanics, 41(1), pp24-45, 2004.

[3] R. Ataur, Y. Azmi, M. Zohadie, D. Ahmad, and W. Ishak, "Simulated Steerability Of A Segmented Rubber Tracked Vehicle During Turning On Sepang Peat Terrain in Malaysia", Int. J. of Heavy Vehicle Systems (IJHVS), Inderscience Publisher, UK: Vol.12, No. 2, pp. 139-168, 2005a.

[4] R. Ataur, Y. Azmi, M. Zohadie, D. Ahmad, and W. Ishak “ Design and Development of a Segmented Rubber Tracked Vehicle for Sepang Peat Terrain in Malaysia", Int. J. of Heavy Vehicle Systems (IJHVS), Inderscience Publisher, UK: Vol.12 No.3, 2005b 
[5] Ataur, R., Azmi, Y., Zohadie, M. Ishak, W and Ahmad, D. "Design Parameters Optimization Simulation of a Segmented Rubber Tracked Vehicle for Sepang Peat in Malaysia", American Journal of Applied Science, Science Publications, New York, USA: Vol.2(3), pp.655-671, 2005c.

[6] H.S. Ooi "Performance of Modified Kubota Carrier RC20P and Porter P6-121 on peat soil", MARDI Report no.110, 1986.

[7] J.Y. Wong "Optimization of design parameters of rigid-link track systems using an advanced computer aided method", Proc. Instn. Mech. Engrs, 208(D), pp153-167, 1998. 